\title{
Fast homoclinic solutions for a class of ordinary $p$-Laplacian systems
}

Bo Du*

\author{
"Correspondence: \\ dubo7307@163.com \\ Department of Mathematics, \\ Huaiyin Normal University, Huaian, \\ Jiangsu 223300, P.R. China
}

\begin{abstract}
We study the existence of fast homoclinic solutions for a kind of ordinary $p$-Laplacian system

$(\mathrm{FHS}) \quad \frac{d}{d t}\left(|\dot{u}(t)|^{p-2} \dot{u}(t)\right)+c|\dot{u}(t)|^{p-2} \dot{u}(t)+\nabla F(t, u)=0$,

where $u \in \mathbb{R}^{n}, 1<p \leq 2$ and $c \geq 0$ are constants and $F(t, u) \in C^{1}\left(\mathbb{R} \times \mathbb{R}^{n}, \mathbb{R}\right)$.

Furthermore, in some particular case, the uniqueness of fast homoclinic solutions of (FHS) is also obtained. Recent results in the literature are generalized and improved.
\end{abstract}

MSC: $60 \mathrm{H} 15 ; 34 \mathrm{G} 20 ; 60 J 65 ; 60 J 75$

Keywords: homoclinic solutions; $p$-Laplacian; critical point

\section{Introduction}

In papers [1] and [2], Arias et al. firstly introduced the concept of fast heteroclinic solutions for the following second-order ODE:

$$
u^{\prime \prime}+c u+f(u)=0
$$

satisfying the boundary conditions

$$
\lim _{t \rightarrow-\infty} u(t)=-1, \quad \lim _{t \rightarrow+\infty} u(t)=0
$$

and obtained the existence results to fast heteroclinic solutions of (1.1) by solving a minimum or constrained minimum problem. Let

$$
\begin{aligned}
& H_{c}=\left\{u \in H_{\mathrm{loc}}^{1}(0,+\infty): \int_{0}^{+\infty} e^{c t} u^{\prime}(t)^{2} d t<+\infty, u(+\infty)=0\right\}, \\
& F(u)=\int_{0}^{u} f(s) d s
\end{aligned}
$$

and

$$
\mathcal{F}: H_{c} \rightarrow \mathbb{R}, \quad \mathcal{F}(u)=\int_{0}^{+\infty} e^{c t}\left(\frac{u^{\prime}(t)^{2}}{2}-F(u(t))\right) d t .
$$

(c) 2015 Du. This article is distributed under the terms of the Creative Commons Attribution 4.0 International License (http://creativecommons.org/licenses/by/4.0/), which permits unrestricted use, distribution, and reproduction in any medium, provided you give appropriate credit to the original author(s) and the source, provide a link to the Creative Commons license, and indicate if changes were made. 
Under some proper conditions, a critical point of $\mathcal{F}$ is a solution of (1.1). It should be a fast solution because of its integrability property near $+\infty$. Motivated by the above works, in a very recent paper, the authors [3] considered a second order non-autonomous system and proved the following main theorem.

Theorem 1.1 Assume that $L$ and $U$ satisfy the following conditions:

$\left(\mathrm{A}_{1}\right) L(t) \in C\left(\mathbb{R}, \mathbb{R}^{n^{2}}\right)$ is a symmetric matrix for all $t \in \mathbb{R}$ and there is a continuous function $\alpha: \mathbb{R} \rightarrow \mathbb{R}$ such that $\alpha(t)>0$ for all $t \in \mathbb{R}$ and $(L(t) q, q) \geq \alpha(t)|q|^{2}$ and $\alpha(t) \rightarrow+\infty$ as $|t| \rightarrow+\infty$

$\left(\mathrm{A}_{2}\right) W(t, q)=a(t)|q|^{\gamma}$, where $a: \mathbb{R} \rightarrow \mathbb{R}$ is a continuous function such that $a\left(t_{1}\right)>0$ for some $t_{1} \in \mathbb{R}$ and $a \in L^{\frac{2}{2-\gamma}}\left(e^{c t}\right), 1<\gamma<2$ is a constant.

Then

$$
\ddot{q}+c \dot{q}+U_{q}(t, q)=0
$$

has at least one nontrivial fast homoclinic solution, where $U(t, q)=-\frac{1}{2}(L(t) q, q)+W(t, q)$. If $c=0,\left(\mathrm{~A}_{1}\right)$ and $\left(\mathrm{A}_{2}\right)$ are supposed, then (1.2) has at least one nontrivial homoclinic solution.

After that, when the coefficient $c$ of (1.2) is variable, Chen et al. [4] dealt with the existence and multiplicity of homoclinic solutions of the following damped vibration problem:

$$
u^{\prime \prime}(t)+q(t) u^{\prime}(t)-L(t) u(t)+\nabla W(t, u(t))=0 .
$$

For more problems of homoclinic solutions, see [5-8]. Motivated by the works mentioned above, when $F$ is the form of (1.3), we will study the existence of homoclinic solutions for the following ordinary $p$-Laplacian system:

$$
\frac{d}{d t}\left(|\dot{u}(t)|^{p-2} \dot{u}(t)\right)+c|\dot{u}(t)|^{p-2} \dot{u}(t)+\nabla F(t, u)=0,
$$

where $1<p \leq 2$ and $c \geq 0$ are constants, $F(t, u) \in C^{1}\left(\mathbb{R} \times \mathbb{R}^{n}, \mathbb{R}\right)$. When $p=2$ and $c=0$, (FHS) reduces to the following second-order Hamiltonian system:

$$
\ddot{u}(t)+\nabla F(t, u)=0 .
$$

Suppose that $F$ is of the form

$$
F(t, u)=-\frac{1}{2}(L(t) u, u)+W(t, u)
$$

where $L(t) \in C\left(\mathbb{R}, \mathbb{R}^{n^{2}}\right)$ is a symmetric matrix for all $t \in \mathbb{R}, W(t, u) \in C^{1}\left(\mathbb{R} \times \mathbb{R}^{n}, \mathbb{R}\right)$. The existence of homoclinic solutions of (HS) has been studied by many authors, we refer the reader to [9-14] and the references therein. In recent years, there have been a few papers [15-18] discussing homoclinic solutions and periodic solutions for second-order systems with a $p$-Laplacian. However, as far as the authors know, there are fewer results of fast homoclinic solutions for (FHS). In the present paper, by using critical point theory, we will obtain some existence results of fast homoclinic solutions for (FHS). Now, we consider two scenarios on $L$ as follows. 
(I) $L(t) \in C\left(\mathbb{R}, \mathbb{R}^{n^{2}}\right)$ is a symmetric matrix for all $t \in \mathbb{R}$ and there is a continuous function $\alpha: \mathbb{R} \rightarrow \mathbb{R}$ such that $\alpha(t)>0$ for all $t \in \mathbb{R}$ and $(L(t) q, q) \geq \alpha(t)|q|^{p}$ and $\alpha(t) \rightarrow+\infty$ as $|t| \rightarrow+\infty$.

Here and subsequently, $(\cdot, \cdot)$ denotes the standard inner product and $|\cdot|$ is the induced norm in $\mathbb{R}^{n}$.

Remark 1.1 Since the $p$-Laplacian operator $\frac{d}{d t}\left(|\dot{u}(t)|^{p-2} \dot{u}(t)\right)$ has stronger nonlinearity, so system (FHS) is more complicated than the corresponding ones of $[3,4]$. In order to overcome these difficulties, we may develop some new techniques in this paper.

We say that a solution $u(t)$ of (FHS) is homoclinic (to 0 ) if $u \in C^{2}\left(\mathbb{R}, \mathbb{R}^{n}\right), u(t) \rightarrow 0$ and $\dot{u}(t) \rightarrow 0$ as $|t| \rightarrow \infty$. If $u(t) \neq 0, u(t)$ is called one nontrivial homoclinic solution. In order to introduce the concept of the fast homoclinic solutions of (FHS), we described some properties of the weighted Sobolev space $E_{c, p}$ on which the certain variational associated with (FHS) is defined and the fast homoclinic solutions are minimizers of the certain functional. For $c \geq 0$, we define the weighted Sobolev space $E_{c, p}$ as follows:

$$
E_{c, p}=\left\{u \in H^{1, p}\left(\mathbb{R}, \mathbb{R}^{n}\right): \int_{\mathbb{R}} e^{c t}\left[\frac{1}{p}|\dot{u}(t)|^{p}+\frac{1}{2}(L(t) u(t), u(t))\right] d t<+\infty\right\} .
$$

Then, for the case that $L$ satisfies (I), $E_{c, p}(c \geq 0)$ is a Hilbert space with the inner product

$$
(x, y)=\int_{\mathbb{R}} e^{c t}\left[\frac{1}{p}(\dot{x}(t), \dot{y}(t))^{\frac{p}{2}}+\frac{1}{2}(L(t) x(t), y(t))\right] d t
$$

and the corresponding norm $\|x\|_{E_{c, p}}^{2}=(x, x)$. On the other hand, for any $u \in H^{1, p}\left(\mathbb{R}, \mathbb{R}^{n}\right)$, $c>0$, satisfying

$$
\int_{\mathbb{R}} e^{c t}\left[|\dot{q}(t)|^{p}+|q(t)|^{p}\right] d t<+\infty
$$

Here, $H^{1, p}\left(\mathbb{R}, \mathbb{R}^{n}\right)$ is a Banach space of functions on $\mathbb{R}$ with values in $\mathbb{R}^{n}$ and the norm

$$
\|u\|_{H^{1, p}}=\left(\|u\|_{p}^{p}+\|\dot{u}\|_{p}^{p}\right)^{\frac{1}{2}} .
$$

We also denote by $L^{p}\left(e^{c t}\right)(1<p \leq 2)$ a Banach space of functions on $\mathbb{R}$ with values in $\mathbb{R}^{n}$ and the norm

$$
\|u\|_{p}=\left(\int_{\mathbb{R}} e^{c t}|u(t)|^{p} d t\right)^{\frac{1}{p}}
$$

For $c \geq 0$, it is obvious that $E_{c, p} \subset L^{p}\left(e^{c t}\right)$ with the embedding being continuous.

Remark 1.2 If $L$ satisfies (I), then there exists a constant $\beta>0$ such that

$$
0.5 \beta\|x\|_{p}^{p} \leq\|x\|_{E_{c, p}}^{2}
$$

where $\beta=\min \{\alpha(t), t \in \mathbb{R}\}$. 
Now we can give the definition of fast homoclinic solution of (FHS).

Definition 1.1 For $c>0$, a homoclinic solution $u$ of (FHS) is called one fast homoclinic solution if $u \in E_{c, p}$.

In this paper we are mainly interested in the case where $W(t, q)$ is of the form $W(t, q)=$ $a(t) V(q)$. Now we present the basic hypothesis on $W$.

(U) $W(t, q)=a(t)|q|^{\gamma}$, i.e., $V(q)=|q|^{\gamma}$, where $a: \mathbb{R} \rightarrow \mathbb{R}$ is a continuous function such that $a\left(t_{1}\right)>0$ for some $t_{1} \in \mathbb{R}$ and $a \in L^{\frac{2}{2-\gamma}}\left(e^{c t}\right)$ and $a \in L^{\frac{2 p}{2-p}}\left(e^{c t}\right)$ with the norm $\|a\|_{\frac{2}{2-\gamma}}$ and $\|a\|_{\frac{2 p}{2-p}}$, respectively, where $\gamma, p \in(1,2)$.

$\left(\mathrm{U}^{\prime}\right) W(t, q)=a(t) V(q)$, where $a: \mathbb{R} \rightarrow \mathbb{R}$ is a continuous function such that $a\left(t_{2}\right)>0$ for some $t_{2} \in \mathbb{R}$ and $a \in L^{p}\left(e^{c t}\right), V \in C^{1}\left(\mathbb{R}^{n}, \mathbb{R}\right), V(0)=0$. Moreover, there exist constants $A_{1}, A_{0}>0,1<\theta<2$ and $0<r \leq 1$ such that

$$
V(q) \geq A_{1}|q|^{\theta}, \quad q \in \mathbb{R}^{n},|q| \leq r
$$

and

$$
\left|V_{q}(q)\right| \leq A_{0}, \quad q \in \mathbb{R}^{n}
$$

Now we state our main results in this paper.

Theorem 1.2 If $c>0$ and suppose that (I) and (U) are satisfied, then (FHS) has at least one nontrivial fast homoclinic solution. If $c=0$, (I) and (U) are satisfied, then (FHS) has at least one nontrivial homoclinic solution. In addition, if there exist positive constants $M_{0}$ and $\omega$ such that

$$
\sup _{t \in \mathbb{R}}|a(t)| \leq M_{0}, \quad\left\|V_{q q}(q)\right\|_{M} \leq \omega \quad \text { for all } q \in \mathbb{R}^{n}
$$

with $M_{0} \omega<1$, where $\left\|V_{q q}(\cdot)\right\|_{M}$ denotes matrix norm, then (FHS) has one and only one nontrivial fast homoclinic solution.

Theorem 1.3 If $c>0$ and suppose that (I) and ( $\left.\mathrm{U}^{\prime}\right)$ are satisfied, then (FHS) has at least one nontrivial fast homoclinic solution. If $c=0$, (I) and $\left(\mathrm{U}^{\prime}\right)$ are satisfied, then (FHS) has at least one nontrivial homoclinic solution.

\section{Preliminaries and main lemmas}

In this section, we give some preliminary results to establish the proofs of our main results. We firstly establish the following compact embedding lemma. Although the proof of the lemma is similar to [3] and [14], for the reader's convenience, we give the details of its proof.

Lemma 2.1 Suppose that $L$ satisfies (I), then the embedding of $E_{c, p}$ in $L^{p}\left(e^{c t}\right)$ is compact.

Proof Let $\left\{u_{k}\right\} \subset E_{c, p}$ be a sequence such that $u_{k} \rightarrow u$ in $E_{c, p}$. We show that $u_{k} \rightarrow u$ in $L^{p}\left(e^{c t}\right)$. Without loss of generality, assume that $u_{k} \rightarrow 0$ in $E_{c, p}$. The Banach-Steinhaus the- 
orem implies that

$$
A=\sup _{k}\left\|u_{k}\right\|_{E_{c, p}}<+\infty
$$

From the properties of $\alpha(t)$, for any $\varepsilon>0$, there is $T_{0}<0$ such that $\frac{1}{\alpha(t)} \leq \varepsilon$ for $t<T_{0}$. Similarly, there is $T_{1}>0$ such that $\frac{1}{\alpha(t)} \leq \varepsilon$ for $t>T_{1}$. Furthermore, $\alpha(t) \geq \min _{t \in\left[T_{0}, T_{1}\right]} \alpha(t)>0$. Let $\left[T_{0}, T_{1}\right]=\Omega$, the operator defined by $S: E_{c, p} \rightarrow H^{1, p}\left(\Omega, \mathbb{R}^{n}\right)$ is a linear continuous map. So $u_{k} \rightarrow 0$ in $H^{1, p}\left(\Omega, \mathbb{R}^{n}\right)$. By the Sobolev theorem, $u_{k} \rightarrow 0$ uniformly on $\Omega$.

- Case 1: $1<p<2$. From the above proof, there is $k_{0}$ such that

$$
\int_{\Omega} e^{c t}\left|u_{k}\right|^{p} d t \leq \varepsilon \quad \text { for } k>k_{0}
$$

On the other hand, we have

$$
\begin{aligned}
\int_{-\infty}^{T_{0}} e^{c t}\left|u_{k}\right|^{p} d t & \leq \varepsilon \int_{-\infty}^{T_{0}} \alpha(t) e^{c t}\left|u_{k}\right|^{p} d t \\
& \leq 2 \varepsilon \int_{-\infty}^{T_{0}} \frac{1}{2} e^{c t}\left(L(t) u_{k}(t), u_{k}(t)\right) d t \\
& \leq 2 \varepsilon A^{2}
\end{aligned}
$$

and

$$
\int_{T_{1}}^{+\infty} e^{c t}\left|u_{k}\right|^{p} d t \leq 2 \varepsilon A^{2}
$$

Combining (2.1)-(2.3), we get $u_{k} \rightarrow 0$ in $L^{p}\left(e^{c t}\right)$.

- Case 2: $p=2$. The proof is similar to [3], we omit it.

Lemma 2.2 Suppose that $I$ and $U$ are satisfied. If $u_{k} \rightarrow u$ in $E_{c, p}$, then $\nabla W\left(t, u_{k}\right) \rightarrow$ $\nabla W(t, u)$ in $L^{p}\left(e^{c t}\right)$.

Proof $\bullet$ Case 1: $p=2$. Assume that $u_{k} \rightarrow u$ in $E_{c, 2}$. We have

$$
\begin{aligned}
\left|\nabla W\left(t, u_{k}\right)-\nabla W(t, u)\right| & =\left.|\gamma a(t)| u_{k}\right|^{\gamma-2} u_{k}-\gamma a(t)|u|^{\gamma-2} u \mid \\
& \leq \gamma|a(t)|\left(\left|u_{k}\right|^{\gamma-1}+|u|^{\gamma-1}\right)
\end{aligned}
$$

which yields that

$$
\left|\nabla W\left(t, u_{k}\right)-\nabla W(t, u)\right|^{2} \leq 2 \gamma^{2}|a(t)|^{2}\left(\left|u_{k}\right|^{2 \gamma-2}+|u|^{2 \gamma-2}\right) .
$$

Multiplying $e^{c t}$ on the both sides of (2.4) and integrating them on $\mathbb{R}$, by (1.4) we have

$$
\begin{aligned}
& \int_{\mathbb{R}} e^{c t}\left|\nabla W\left(t, u_{k}\right)-\nabla W(t, u)\right|^{2} d t \\
& \quad \leq 2 \gamma^{2} \int_{\mathbb{R}} e^{c t}|a(t)|^{2}\left(\left|u_{k}\right|^{2 \gamma-2}+|u|^{2 \gamma-2}\right) d t
\end{aligned}
$$




$$
\begin{aligned}
& \leq 2 \gamma^{2}\left(\int_{\mathbb{R}} e^{c t}\left|u_{k}\right|^{2} d t\right)^{\gamma-1}\|a\|_{\frac{2}{2-\gamma}}^{2}+2 \gamma^{2}\left(\int_{\mathbb{R}} e^{c t}|u|^{2} d t\right)^{\gamma-1}\|a\|_{\frac{2}{2-\gamma}}^{2} \\
& \leq 2^{\gamma} \gamma^{2} \beta^{1-\gamma}\left\|u_{k}\right\|_{c, 2}^{2 \gamma-2}\|a\|_{\frac{2}{2-\gamma}}^{2}+2^{\gamma} \gamma^{2} \beta^{1-\gamma}\|a\|_{\frac{2}{2-\gamma}}^{2} \\
& \leq 2^{\gamma} \gamma^{2} \beta^{1-\gamma}\|a\|_{\frac{2}{2-\gamma}}^{2}\left(\left\|u_{k}\right\|_{E_{c, 2}}^{2 \gamma-2}+\|u\|_{E_{c, 2}}^{2 \gamma-2}\right) .
\end{aligned}
$$

From $u_{k} \rightarrow u$ in $E_{c, 2}$ and the Banach-Steinhaus theorem, there is a constant $M_{1}>0$ such that

$$
\left\|u_{k}\right\|_{E_{c, 2}} \leq M_{1}, \quad\|u\|_{E_{c, 2}} \leq M_{1} .
$$

From (2.5) and (2.6), we have

$$
\int_{\mathbb{R}} e^{c t}\left|\nabla W\left(t, u_{k}\right)-\nabla W(t, u)\right|^{2} d t \leq 2^{\gamma+1} \gamma^{2} \beta^{1-\gamma}\|a\|_{22}^{2} M_{1}^{2 \gamma-2} .
$$

From Lemma 2.1, we have

$$
e^{c t} u_{k}(t) \rightarrow e^{c t} u(t) \quad \text { for almost } t \in \mathbb{R},
$$

which yields that $u_{k}(t) \rightarrow u(t)$ for almost $t \in \mathbb{R}$. Then, using Lebesgue's convergence theorem, $\nabla W\left(t, u_{k}\right) \rightarrow \nabla W(t, u)$ in $L^{2}\left(e^{c t}\right)$.

- Case 2: $1<p<2$. Similar to the above proof, we have

$$
\begin{aligned}
& \int_{\mathbb{R}} e^{c t}\left|\nabla W\left(t, u_{k}\right)-\nabla W(t, u)\right|^{p} d t \\
& \quad \leq \gamma^{p} \int_{\mathbb{R}} e^{c t}|a(t)|^{p}\left(\left|u_{k}\right|^{\gamma-1}+|u|^{\gamma-1}\right)^{p} d t \\
& \quad \leq \gamma^{p}\|a\|_{\frac{2 p}{2-p}}^{2-p}\left(\int_{\mathbb{R}} e^{c t}\left(\left|u_{k}\right|^{\gamma-1}+|u|^{\gamma-1}\right)^{2} d t\right)^{\frac{p}{2}} \\
& \quad \leq \gamma^{p}\|a\|_{\frac{2 p}{2-p}}^{2-p}\left(\int_{\mathbb{R}} 2 e^{c t}|a(t)|^{2}\left(\left|u_{k}\right|^{2 \gamma-2}+|u|^{2 \gamma-2}\right) d t\right)^{\frac{p}{2}} .
\end{aligned}
$$

From the proof of Case 1, there exists a constant $M_{2}>0$ such that

$$
\int_{\mathbb{R}} e^{c t}\left|\nabla W\left(t, u_{k}\right)-\nabla W(t, u)\right|^{p} d t \leq M_{2}\|a\|_{\frac{2 p}{2-p}}^{2-p},
$$

since $a \in\|a\|_{\frac{2 p}{2-p}}$. Thus $\nabla W\left(t, u_{k}\right) \rightarrow \nabla W(t, u)$ in $L^{p}\left(e^{c t}\right)$. Hence, combining the proof of Case 1 and Case 2, the proof is completed.

From the proofs of Lemmas 2.1 and 2.2, it is clear that if $u_{k} \rightarrow u$ in $E_{c, p}$, then $\nabla W\left(t, u_{k}\right) \rightarrow$ $\nabla W(t, u)$ in $L^{p}\left(e^{c t}\right)$. In the following we will establish the corresponding variational framework to obtain the existence of homoclinic solutions of (FHS). Define the functional $I: E_{c, p} \rightarrow \mathbb{R}$ by

$$
\begin{aligned}
I(u) & =\int_{\mathbb{R}} e^{c t}\left[\frac{1}{p}|\dot{u}(t)|^{p}+\frac{1}{2}(L(t) u(t), u(t))-W(t, u(t))\right] d t \\
& =\|u\|_{E_{c, p}}^{2}-\int_{\mathbb{R}} e^{c t} W(t, u(t)) d t .
\end{aligned}
$$


Lemma 2.3 Under the conditions of Theorem 1.2, we have

$$
\begin{aligned}
I^{\prime}(u) v= & \int_{\mathbb{R}} e^{c t}\left[\left(|\dot{u}(t)|^{p-2} \dot{u}(t), \dot{v}(t)\right)+(L(t) u(t), v(t))\right. \\
& -(\nabla W(t, u(t)), v(t))] d t, \quad u, v \in E_{c, p} .
\end{aligned}
$$

Moreover, $I \in C^{1}\left(E_{c, p}, \mathbb{R}\right)$, i.e., I is a continuously Fréchet-differentiable functional defined on $E_{c, p}$ and any critical point of I on $E_{c, p}$ is a classic solution of (FHS).

Proof Firstly we show that $I: E_{c, p} \rightarrow \mathbb{R}$. From (1.4) and Hölder's inequality, we have

$$
\begin{aligned}
0 & \leq \int_{\mathbb{R}} e^{c t}|W(t, u(t))| d t=\int_{\mathbb{R}} e^{c t}|a(t) \| u(t)|^{\gamma} d t \\
& \leq\left(\int_{\mathbb{R}}\left(e^{\left(1-\frac{\gamma}{2}\right) c t}|a(t)|\right)^{\frac{2}{2-\gamma}} d t\right)^{\frac{2-\gamma}{2}}\left(\int_{\mathbb{R}}\left(e^{\frac{\gamma}{2} c t}|u(t)|^{\gamma}\right)^{\frac{2}{\gamma}} d t\right)^{\frac{\gamma}{2}} \\
& =\|a\|_{\frac{2}{2-\gamma}}\left(\int_{\mathbb{R}} e^{c t}|u(t)|^{2} d t\right)^{\frac{\gamma}{2}} \\
& \leq 2^{0.5 \gamma} \beta^{-0.5 \gamma}\|a\|_{\frac{2}{2-\gamma}}\left(\int_{\mathbb{R}} e^{c t}|u(t)|^{2} d t\right)^{\frac{\gamma}{2}} \\
& \leq 2^{0.5 \gamma} \beta^{-0.5 \gamma}\|a\|_{\frac{2}{2-\gamma}}\|u\|_{c, p}^{\gamma} .
\end{aligned}
$$

Hence, one has $I: E_{c, p} \rightarrow \mathbb{R}$. Next, we prove that $I \in C^{1}\left(E_{c, p}, \mathbb{R}\right)$. Rewrite $I$ as follows:

$$
I=I_{1}-I_{2}
$$

where

$$
\begin{aligned}
& I_{1}=\int_{\mathbb{R}} e^{c t}\left[\frac{1}{p}|\dot{u}(t)|^{p}+\frac{1}{2}(L(t) u(t), u(t))\right] d t, \\
& I_{2}=\int_{\mathbb{R}} e^{c t} W(t, u(t)) d t .
\end{aligned}
$$

Obviously, $I_{1} \in C^{1}\left(E_{c}, \mathbb{R}\right)$ and

$$
I_{1}^{\prime}(u) v=\int_{\mathbb{R}} e^{c t}\left[\left(|\dot{u}(t)|^{p-2} \dot{u}(t), \dot{v}(t)\right)+(L(t) u(t), v(t))\right] d t .
$$

Now, we will show

$$
I_{2}^{\prime}(u) v=\int_{\mathbb{R}} e^{c t}(\nabla W(t, u(t)), v(t)) d t .
$$

Let us define $J(u): E_{c, p} \rightarrow \mathbb{R}$ as follows:

$$
J(u) v=\int_{\mathbb{R}} e^{c t}(\nabla W(t, u(t)), v(t)) d t, \quad v \in E_{c, p} .
$$


Obviously, $J(u)$ is linear. Now we show that $J(u)$ is bounded. For each $u \in E_{c, p}$, by (1.4) and Hölder's inequality, we have

$$
\begin{aligned}
|J(u) v|= & \left|\int_{\mathbb{R}} e^{c t}(\nabla W(t, u(t)), v(t)) d t\right| \leq \int_{\mathbb{R}} e^{c t}|a(t)||u(t)|^{\gamma-1}|v(t)| d t \\
\leq & \left(\int_{\mathbb{R}} e^{c t}|u(t)|^{2 \gamma-2}|a(t)|^{2} d t\right)^{\frac{1}{2}}\left(\int_{\mathbb{R}} e^{c t}|v(t)|^{2} d t\right)^{\frac{1}{2}} \\
\leq & \left(\int_{\mathbb{R}}\left(e^{(2-\gamma) c t}|a(t)|^{2}\right)^{\frac{1}{2-\gamma}} d t\right)^{\frac{2-\gamma}{2}}\left(\int_{\mathbb{R}}\left(e^{(\gamma-1) c t}|u(t)|^{2 \gamma-2}\right)^{\frac{1}{\gamma-1}} d t\right)^{\frac{\gamma-1}{2}} \\
& \times\left(\int_{\mathbb{R}} e^{c t}|v(t)|^{2} d t\right)^{\frac{1}{2}} \\
= & \left(\int_{\mathbb{R}} e^{c t}|a(t)|^{\frac{2}{2-\gamma}} d t\right)^{\frac{2-\gamma}{2}}\left(\int_{\mathbb{R}} e^{c t}|u(t)|^{2} d t\right)^{\frac{\gamma-1}{2}}\left(\int_{\mathbb{R}} e^{c t}|v(t)|^{2} d t\right)^{\frac{1}{2}} \\
\leq & \|a\|_{\frac{2}{2-\gamma}} 2^{0.5 \gamma(\gamma-1)} \beta^{-0.5 \gamma(\gamma-1)}\|u\|_{E_{c, p}}^{\gamma-1} 2^{0.5 \gamma} \beta^{-0.5 \gamma}\|v\|_{E_{c, p}} \\
= & \|a\|_{\frac{2}{2-\gamma}} 2^{0.5 \gamma^{2}} \beta^{-0.5 \gamma^{2}}\|u\|_{E_{c, p}}^{\gamma-1}\|v\|_{E_{c, p}}<+\infty .
\end{aligned}
$$

Moreover, for $u$ and $v \in E_{c, p}$, by the mean value theorem, we have

$$
\begin{gathered}
\int_{\mathbb{R}} e^{c t} W(t, u(t)+v(t)) d t-\int_{\mathbb{R}} e^{c t} W(t, u(t)) d t \\
=\int_{\mathbb{R}} e^{c t}(\nabla W(t, u(t)+h(t) v(t)), v(t)) d t,
\end{gathered}
$$

where $h(t) \in(0,1)$. Hence, by Lemma 2.2 and Hölder's inequality, we have

$$
\begin{aligned}
& \int_{\mathbb{R}} e^{c t}(\nabla W(t, u(t)+h(t) v(t)), v(t)) d t-\int_{\mathbb{R}} e^{c t}(\nabla W(t, u(t)), v(t)) d t \\
& \quad \rightarrow 0 \quad \text { as }\|v\|_{E_{c, p}} \rightarrow 0
\end{aligned}
$$

From Lemma 2.2, we see that (2.9) holds. It remains to prove that $I_{2}^{\prime}$ is continuous. Suppose that $u \rightarrow u_{0}$ in $E_{c, p}$ and note that

$$
I_{2}^{\prime}(u) v-I_{2}^{\prime}\left(u_{0}\right) v=\int_{\mathbb{R}} e^{c t}\left(\nabla W(t, u(t))-\nabla W\left(t, u_{0}(t)\right), v(t)\right) d t .
$$

By Lemma 2.2, we have

$$
I_{2}^{\prime}(u) v-I_{2}^{\prime}\left(u_{0}\right) v \rightarrow 0 \quad \text { as } u \rightarrow u_{0}
$$

and $I \in C^{1}\left(E_{c, p}, \mathbb{R}\right)$. On the other hand, if $u$ is a critical point of $I$, by (2.9), we know that $e^{c t}[L(t) u-\nabla W(t, u)]$ is the weak derivative $e^{c t}|\dot{u}(t)|^{p-2} \dot{u}(t)$, and $\dot{u}$ and $\ddot{u}$ are continuous. It is easy to check that critical points of $I$ on $E_{c, p}$ are classic solutions satisfying $u(t) \rightarrow 0$ and $\dot{u}(t) \rightarrow 0$ as $|t| \rightarrow \infty$.

Lemma 2.4 Under the conditions of Theorem 1.2, we have that (2.8) holds and I $\in$ $C^{1}\left(E_{c, p}, \mathbb{R}\right)$. Moreover, any critical point of I on $E_{c, p}$ is a classic homoclinic solution of (FHS). 
Proof Since the proofs are similar to the proofs of Lemma 2.2 and Lemma 2.3, so we omit the details of their proofs.

Lemma 2.5 Suppose that (I) and $\left(\mathrm{U}^{\prime}\right)$ are satisfied. If $u_{k} \rightarrow u$ in $E_{c, p}$, then $\nabla W\left(t, u_{k}\right) \rightarrow$ $\nabla W(t, u)$ in $L^{p}\left(e^{c t}\right)$.

Proof $\mathrm{By}\left(\mathrm{U}^{\prime}\right)$, we have

$$
\left|\nabla W\left(t, u_{k}\right)-\nabla W(t, u)\right| \leq 2 A_{0}|a(t)|
$$

which yields that

$$
e^{c t}\left|\nabla W\left(t, u_{k}\right)-\nabla W(t, u)\right|^{p} \leq 2^{p} A_{0}^{p} e^{c t}|a(t)|^{p} .
$$

Integrating the above inequality on $\mathbb{R}$ and by Hölder's inequality, we obtain that

$$
\begin{aligned}
\int_{\mathbb{R}} e^{c t}\left|\nabla W\left(t, u_{k}\right)-\nabla W(t, u)\right|^{p} d t & \leq \int_{\mathbb{R}} 2^{p} A_{0}^{p} e^{c t}|a(t)|^{p} d t \\
& \leq 2^{p} A_{0}^{p}\|a\|_{p}^{p}
\end{aligned}
$$

since $a \in L^{p}\left(e^{c t}\right)$. On the other hand, by Lemma 2.1, we have $u_{k}(t) \rightarrow u(t)$ for almost each $t \in \mathbb{R}$. Consequently, using the Lebesgue's convergence theorem, we finish the proof of this lemma.

Lemma 2.6 Under the conditions of Theorem 1.3, we have that (2.8) holds and I $\in$ $C^{1}\left(E_{c, p}, \mathbb{R}\right)$. Moreover, any critical point of I on $E_{c, p}$ is a classical homoclinic solution of (FHS).

Proof From $\left(\mathrm{U}^{\prime}\right)$ and $V(0)=0$, we have

$$
V(u)=\left|\int_{0}^{1} V_{u}(\mu u) u d \mu\right| \leq A_{0}|u| .
$$

By (2.10) and (1.4), we have

$$
\begin{aligned}
0 & \leq \int_{\mathbb{R}} e^{c t}|W(t, u(t))| d t \leq A_{0} \int_{\mathbb{R}} e^{c t}|a(t) \| u(t)| d t \\
& \leq A_{0}\|a\|_{q}\|u\|_{p} \leq A_{0} 2^{1 / p} \beta^{-1 / p}\|a\|_{q}\|u\|_{E_{c, p}}^{2 / p},
\end{aligned}
$$

where $1 / p+1 / q=1$, which together with (2.7) implies that $I: E_{c, p} \rightarrow \mathbb{R}$. Moreover, according to the proof of Lemma 2.3, it is sufficient to show that for any given $u \in E_{c, p}$, the operator $J(u): E_{c, p} \rightarrow \mathbb{R}$ defined as follows:

$$
J(u) v=\int_{\mathbb{R}} e^{c t}(\nabla W(t, u), v) d t, \quad \forall v \in E_{c, p}
$$

is linear and bounded. It is obvious that $J(u)$ is linear. By $\left(\mathrm{U}^{\prime}\right)$, we have

$$
|J(u) v| \leq A_{0} \int_{\mathbb{R}} e^{c t}\left|a(t)\left\|u(t) \mid d t \leq A_{0}\right\| a\left\|_{q}\right\| u\left\|_{p} \leq A_{0} 2^{1 / p} \beta^{-1 / p}\right\| a\left\|_{q}\right\| u \|_{E_{c, p}}^{2 / p},\right.
$$


which implies that $J(u)$ is bounded. The remainder is similar to the proof of Lemma 2.3, so we omit the details of its proof.

In the following, we introduce some necessary definitions and some well-known results in the variational methods and critical point theory (see [19] and [20]). Let $E$ be a real Banach space.

Definition 2.1 ([19]) $I: E \rightarrow \mathbb{R}$ is coercive if $I(u) \rightarrow+\infty$ for $\|u\| \rightarrow+\infty$.

Definition 2.2 ([20]) $I \in C^{1}(E, \mathbb{R})$ is said to satisfy the (PS) condition if any sequence $\left\{u_{j}\right\}_{j \in \mathbb{N}} \subset E$, for which $\left\{u_{j}\right\}_{j \in \mathbb{N}}$ is bounded and $I^{\prime}\left(u_{j}\right) \rightarrow 0$ as $j \rightarrow+\infty$, possesses a convergent subsequence in $E$.

Lemma 2.7 ([20]) Let E be a real Banach space and $I \in C^{1}(E, \mathbb{R})$ satisfying the $(P S)$ condition. If $I$ is bounded from below, then $c_{0}=\inf _{E} I$ is a critical point of $I$.

Lemma 2.8 ([19]) If $I: E \rightarrow \mathbb{R}$ is differentiable, every local minimum (maximum) point $u$ satisfies the Euler equation $I^{\prime}(u)=0$.

\section{The proof of main results}

In order to use Lemmas 2.7 and 2.8 for the proofs of Theorems 1.2 and 1.3, we firstly show that $I$ satisfies the (PS) condition.

Lemma 3.1 Under the conditions of Theorem 1.2, I satisfies the (PS) condition.

Proof Assume that $\left\{u_{j}\right\}_{j \in \mathbb{N}} \subset E_{c, p}$ is a sequence that $\left\{I\left(u_{j}\right)\right\}_{j \in \mathbb{N}}$ is bounded and $I^{\prime}\left(u_{j}\right) \rightarrow 0$ as $j \rightarrow+\infty$. Then there exists a constant $M_{3}>0$ such that

$$
\left|I\left(u_{j}\right)\right| \leq M_{3} \quad \text { for each } j \in \mathbb{N} \text {. }
$$

We claim that $\left\{u_{j}\right\}_{j \in \mathbb{N}}$ is bounded in $E_{c, p}$. In fact, from (1.4), (2.7), (3.1) and (U), we have

$$
\begin{aligned}
\left\|u_{j}\right\|_{E_{c, p}}^{2} & =I\left(u_{j}\right)+\int_{\mathbb{R}} e^{c t} W\left(t, u_{j}(t)\right) d t \\
& \leq M_{3}+\int_{\mathbb{R}} e^{c t}\left|a(t) \| u_{j}(t)\right|^{\gamma} d t \\
& \leq M_{3}+2^{0.5 \gamma} \beta^{-0.5 \gamma}\|a\|_{2-\gamma}\left(\int_{\mathbb{R}} e^{c t}\left|u_{j}(t)\right|^{2} d t\right)^{\frac{\gamma}{2}} \\
& \leq M_{3}+2^{0.5 \gamma} \beta^{-0.5 \gamma}\|a\|_{\frac{2}{2-\gamma}}\left\|u_{j}\right\|_{E_{c, p}}^{\gamma} .
\end{aligned}
$$

Since $1<\gamma<2$, (3.2) implies that $\left\{u_{j}\right\}_{j \in \mathbb{N}}$ is bounded in $E_{c, p}$. By use of Lemma 2.1, the sequence $\left\{u_{j}\right\}_{j \in \mathbb{N}}$ has a subsequence, again denoted by $\left\{u_{j}\right\}_{j \in \mathbb{N}}$, and there is $u \in E_{c, p}$ such that

$$
\begin{array}{ll}
u_{j} \rightarrow u & \text { weakly in } E_{c, p}, \\
u_{j} \rightarrow u & \text { strongly in } L^{p}\left(e^{c t}\right) .
\end{array}
$$


Thus

$$
\left(I^{\prime}\left(u_{j}\right)-I^{\prime}(u)\right)\left(u_{j}-u\right) \rightarrow 0
$$

and by Lemma 2.2 we have

$$
\int_{\mathbb{R}} e^{c t}\left(\nabla W\left(t, u_{j}(t)\right)-\nabla W(t, u(t)), u_{j}(t)-u(t)\right) d t \rightarrow 0, \quad j \rightarrow+\infty .
$$

On the other hand, from

$$
\left\|u_{j}-u\right\|_{E_{c, p}}^{2}=\left(I^{\prime}\left(u_{j}\right)-I^{\prime}(u),\left(u_{j}-u\right)\right)+\int_{\mathbb{R}} e^{c t}\left(\nabla W\left(t, u_{j}(t)\right)-\nabla W(t, u(t)), u_{j}(t)-u(t)\right) d t,
$$

we have

$$
\left\|u_{j}-u\right\|_{E_{c, P}}^{2} \rightarrow 0 \quad \text { as } j \rightarrow+\infty
$$

Thus $\left\{u_{j}\right\}_{j \in \mathbb{N}}$ converges strongly to $u$ in $E_{c, p}$.

Note that there is no difference between the proofs of the fast homoclinic solutions and the usual homoclinic solutions. So, in the following proofs of our main results, we do not consider them for $c>0$ and $c=0$, respectively.

Proof of Theorem 1.2 From (1.4), (2.7) and (U), for every $m \in \mathbb{R} \backslash\{0\}$ and $u \in E_{c, p} \backslash\{0\}$, we have

$$
\begin{aligned}
I(m u) & =m^{2}\|u\|_{E_{c, p}}^{2}-\int_{\mathbb{R}} e^{c t} W(t, m u(t)) d t \\
& \geq m^{2}\|u\|_{E_{c, p}}^{2}-|m|^{\gamma} \int_{\mathbb{R}} e^{c t}|a(t) \| u(t)|^{\gamma} d t \\
& \geq m^{2}\|u\|_{E_{c, p}}^{2}-|m|^{\gamma} 2^{0.5 \gamma} \beta^{-0.5 \gamma}\|a\|_{\frac{2}{2-\gamma}}\left(\int_{\mathbb{R}} e^{c t}|u(t)|^{2} d t\right)^{\frac{\gamma}{2}} \\
& \geq m^{2}\|u\|_{E_{c, p}}^{2}-|m|^{\gamma} 2^{0.5 \gamma} \beta^{-0.5 \gamma}\|a\|_{\frac{2}{2-\gamma}}\|u\|_{E_{c, p}}^{\gamma} .
\end{aligned}
$$

Since $1<\gamma<2$, (3.3) implies that $I(m u) \rightarrow+\infty$ as $|m| \rightarrow+\infty$. Hence, $I$ is bounded from below. From Lemmas 2.7 and 3.1, I possesses a critical value $c_{0}=\inf _{u \in E_{c, p}} I(u)$ and there is a critical point $u \in E_{c, p}$ such that $I(u)=c_{0}$ and $I^{\prime}(u)=0$. In the following, we show that the critical point obtained above $u \neq 0$. By (U), there is $\delta_{1}>0$ such that $a(t)>0$ for any $t \in\left[t-\delta_{1}, t+\delta_{1}\right]$. Take $c_{1} \in \mathbb{R}^{n}$ with $\left|c_{1}\right| \neq 0$, and let $\phi \in E_{c, p}$ be defined by

$$
\phi(t)= \begin{cases}c_{1} \cos \left[\frac{\pi}{2 \delta_{1}}\left(t-t_{1}\right)\right], & t \in\left[t_{1}-\delta_{1}, t_{1}+\delta_{1}\right], \\ 0, & t \in \mathbb{R} \backslash\left[t_{1}-\delta_{1}, t_{1}+\delta_{1}\right] .\end{cases}
$$

Thus, by (2.7),

$$
I(m \phi)=m^{2}\|\phi\|_{E_{c}}^{2}-|m|^{\gamma} \int_{t_{1}-\delta_{1}}^{t_{1}+\delta_{1}} e^{c t} a(t)|\phi(t)|^{\gamma} d t
$$


which yields that $I(m \phi)<0$ for $|m|$ small enough since $1<\gamma<2$. Hence, the critical point $u \in E_{c, p}$ is nontrivial. Finally, we show that if (1.5) holds, then (FHS) has one and only one nontrivial homoclinic solution. Suppose by contradiction that there would be at least two different homoclinic solutions $q_{1}$ and $q_{2}$. From Lemma 2.3 and (1.5), we have

$$
\begin{aligned}
0 & =\left(I^{\prime}\left(q_{1}\right)-I^{\prime}\left(q_{2}\right), q_{1}-q_{2}\right) \\
& =\left\|q_{1}-q_{2}\right\|_{E_{c, p}}^{2}+\int_{\mathbb{R}} e^{c t}\left(a(t) V_{q}\left(q_{1}\right)-a(t) V_{q}\left(q_{2}\right), q_{1}(t)-q_{2}(t)\right) d t \\
& =\left\|q_{1}-q_{2}\right\|_{E_{c, p}}^{2}+\int_{\mathbb{R}} e^{c t}\left(a(t) V_{q q}\left(q_{3}\right)\left(q_{1}-q_{2}\right), q_{1}(t)-q_{2}(t)\right) d t \\
& \geq\left\|q_{1}-q_{2}\right\|_{E_{c, p}}^{2}-M_{0}\left\|V_{q q}\left(q_{3}\right)\right\|_{M}\left\|q_{1}-q_{2}\right\|_{E_{c, p}}^{2} \\
& \geq\left\|q_{1}-q_{2}\right\|_{E_{c, p}}^{2}-M_{0} \omega\left\|q_{1}-q_{2}\right\|_{E_{c, p}}^{2} .
\end{aligned}
$$

Since $M_{0} \omega<1$, we get $\left\|q_{1}-q_{2}\right\|_{E_{c, p}}=0$ and $q_{1}(t) \equiv q_{2}(t)$ for all $t \in \mathbb{R}$.

Lemma 3.2 Under the conditions of Theorem 1.3, I satisfies the (PS) condition.

Proof It is sufficient to show that for any sequence $\left\{u_{j}\right\}_{j \in \mathbb{N}} \subset E_{c, p}$ such that $\left\{I\left(u_{j}\right)\right\}_{j \in \mathbb{N}}$ is bounded, $I^{\prime}\left(u_{j}\right) \rightarrow 0$ as $j \rightarrow+\infty$, then $\left\{u_{j}\right\}_{j \in \mathbb{N}}$ is bounded in $E_{c, p}$. Since $\left\{I\left(u_{j}\right)\right\}_{j \in \mathbb{N}}$ is bounded, there exists constant $B_{1}>0$ such that

$$
\left|I\left(u_{j}\right)\right| \leq B_{1} \quad \text { for each } j \in \mathbb{N} \text {. }
$$

From (1.4) and (3.4), we have

$$
\left\|u_{j}\right\|_{E_{c, p}}^{2}=I\left(u_{j}\right)+\int_{\mathbb{R}} e^{c t} W\left(t, u_{j}(t)\right) d t \leq B_{1}+A_{0}\|a\|_{q}\left\|u_{j}\right\|_{p} \leq A_{0} 2^{1 / p} \beta^{-1 / p}\|a\|_{q}\left\|u_{j}\right\|_{E_{c, p}}^{2 / p},
$$

which implies that $\left\{u_{j}\right\}_{j \in \mathbb{N}}$ is bounded in $E_{c, p}$.The remainder is similar to the proof of Lemma 3.1, so we omit the details of its proof.

Proof of Theorem 1.3 From (1.4), (2.7) and ( $\left.\mathrm{U}^{\prime}\right)$, for every $m \in \mathbb{R} \backslash\{0\}$ and $u \in E_{c, p} \backslash\{0\}$, we have

$$
\begin{aligned}
I(m u) & =m^{2}\|u\|_{E_{c, p}}^{2}-\int_{\mathbb{R}} e^{c t} W(t, m u(t)) d t \\
& \geq m^{2}\|u\|_{E_{c, p}}^{2}-A_{0} 2^{1 / p} \beta^{-1 / p}|m|\|a\|_{q}\|u\|_{E_{c, p},}^{2 / p},
\end{aligned}
$$

which $I(m u) \rightarrow+\infty$ as $|m| \rightarrow+\infty$. Consequently, $I$ is a functional bounded from below. By Lemmas 2.7 and 3.2, $I$ possesses a critical value $c_{0}=\inf _{u \in E_{c, p}} I(u)$, i.e., there is a critical point $u \in E_{c, p}$ such that $I(u)=c_{0}$ and $I^{\prime}(u)=0$. In the following, we show that the critical point obtained above $u \neq \equiv 0$. By $\left(\mathrm{U}^{\prime}\right)$, there is $\delta_{2}>0$ such that $a(t)>0$ for any $t \in\left[t-\delta_{2}, t+\right.$ $\left.\delta_{2}\right]$. Take $c_{2} \in \mathbb{R}^{n}$ with $\left|c_{2}\right|=r$, where $r$ is defined in $\left(\mathrm{U}^{\prime}\right)$ and let $\phi \in E_{c, p}$ be defined by

$$
\phi(t)= \begin{cases}c_{2} \cos \left[\frac{\pi}{2 \delta_{2}}\left(t-t_{2}\right)\right], & t \in\left[t_{2}-\delta_{2}, t_{2}+\delta_{2}\right], \\ 0, & t \in \mathbb{R} \backslash\left[t_{2}-\delta_{2}, t_{2}+\delta_{2}\right] .\end{cases}
$$


Then $\phi(t) \leq r \leq 1$ and

$$
I(m \phi) \leq m^{2}\|\phi\|_{E_{c, p}}^{2}-A_{1}|m|^{\theta} \int_{t_{1}}^{t_{2}} e^{c t} a(t)|\phi(t)|^{\theta} d t
$$

which yields that $I(m \phi)<0$ for $|m|$ small enough since $1<\theta<2$. The critical point $u \in E_{c, p}$ obtained above is nontrivial.

Remark 3.1 The proofs of Theorems 1.2 and 1.3 are similar to [3], the main difference is structure of the Hilbert space. Obviously, the Hilbert space $E_{c, p}$ in the present paper is more complicated than the corresponding ones of past works [3, 4, 9-14].

\section{Conclusions and further discussion}

In this paper, by using variational method, we have dealt with a kind of ordinary $p$-Laplacian system and obtained the existence of fast homoclinic solutions to the above system. However, we mainly consider the case that $W(t, u)$ is subquadratic as $|u| \rightarrow+\infty$. When $W(t, u)$ is superquadratic as $|u| \rightarrow+\infty$, we only find Chen et al. [4] obtained the existence of fast homoclinic solutions for a class of second order systems. But, for the $p$ Laplacian system, there are few results on the existence of fast homoclinic solutions. We hope that the related results will appear in the near future.

\section{Competing interests}

The author declares that they have no competing interests.

\section{Acknowledgements}

This work is supported by the NNSF (No. 11571136) of China.

Received: 5 August 2015 Accepted: 24 September 2015 Published online: 09 October 2015

References

1. Arias, M, Campos, J, Robles-Péres, AM, Sanchez, L: Fast and heteroclinic solutions for a second order ODE related to Fisher-Kolmogorov's equation. Calc. Var. Partial Differ. Equ. 21, 319-334 (2004)

2. Arias, M: Fast and heteroclinic solutions for a second order ODE. In: Proceedings of the 2005 Oujda International Conference on Nonlinear Analysis. Electron. J. Differ. Equ. Conf., vol. 14, pp. 119-124 (2006)

3. Zhang, Z, Yuan, R: Fast homoclinic solutions for some second order non-autonomous systems. J. Math. Anal. Appl. 376, 51-63 (2011)

4. Chen, P, Tang, X, Agarwal, RP: Fast homoclinic solutions for a class of damped vibration problems. Appl. Math. Comput. 219, 6053-6065 (2013)

5. Lu, S, Lu, M: Homoclinic solutions for a second-order $p$-Laplacian functional differential system with local condition. Adv. Differ. Equ. 2014, 244 (2014)

6. Lu, S: Homoclinic solutions for a nonlinear second order differential system with $p$-Laplacian operator. Nonlinear Anal., Real World Appl. 12, 525-534 (2011)

7. Lu, S: Homoclinic solutions for a class of prescribed mean curvature Liénard equations. Adv. Differ. Equ. 2015, 239 (2015)

8. Lu, S: Existence of homoclinic solutions for a class of neutral functional differential equations. Acta Math. Sin. Engl. Ser. 6, 1261-1274 (2012)

9. Caldiroli, P, Montecchiari, P: Homoclinic orbits for second order Hamiltonian systems with potential changing sign. Commun. Appl. Nonlinear Anal. 1, 97-129 (1994)

10. Coti Zelati, V, Rabinowtiz, PH: Homoclinic orbits for second order Hamiltonian systems possessing superquadratic potential. J. Am. Math. Soc. 4, 693-727 (1991)

11. Ding, $Y$, Lee, C: Homoclinics for asymptotically quadratic and superquadratic Hamiltonian systems. Nonlinear Anal. 71, 1395-1413 (2009)

12. Rabinowtiz, PH: Homoclinic orbits for a class of Hamiltonian systems. Proc. R. Soc. Edinb., Sect. A 114, 33-38 (1990)

13. Rabinowtiz, PH, Tanaka, K: Some results on connecting orbits for a class of Hamiltonian systems. Math. Z. 206, 473-499 (1991)

14. Omana, W, Willem, M: Homoclinic orbits for a class of Hamiltonian systems. Differ. Integral Equ. 5, 1115-1120 (1992)

15. Jebelean, P, Morosanu, G: Ordinary p-Laplacian systems with nonlinear boundary conditions. J. Math. Anal. Appl. 313, 738-753 (2006)

16. LV, H, O'Regan, D, Agarwal, RP: On the existence of multiple periodic solutions for the vector $p$-Laplacian via critical point theory. Appl. Math. 50, 555-568 (2005) 
17. Mansevich, R, Mawhin, J: Periodic solutions for nonlinear systems with $p$-Laplacian-like operators. J. Differ. Equ. 145 367-393 (1998)

18. Mawhin, J: Some boundary value problems for Hartman-type perturbations of the ordinary vector $p$-Laplacian. Nonlinear Anal. 40, 497-503 (2000)

19. Mawhin, J, Willem, M: Critical Point Theory and Hamiltonian Systems. Springer, New York (1989)

20. Rabinowtiz, PH: Minimax Methods in Critical Theory with Applications to Differential Equations. CBMS Reg. Conf. Ser. Math., vol. 65. Am. Math. Soc., Providence (1986)

Submit your manuscript to a SpringerOpen ${ }^{\circ}$ journal and benefit from:

- Convenient online submission

- Rigorous peer review

- Immediate publication on acceptance

- Open access: articles freely available online

- High visibility within the field

- Retaining the copyright to your article 